\title{
Oropharyngeal Kaposi’s Sarcoma from an Immunocompetent Host: A Case Report
}

\author{
Yin-Ping Hsu ${ }^{1}$, Li-Jen Hsin ${ }^{1}$ and Tuan-Jen Fang ${ }^{1,2^{*}}$ \\ ${ }^{1}$ Departments of Otolaryngology-Head \& Neck Surgery, Taiwan \\ ${ }^{2}$ College of Medicine, Chang Gung University, Taiwan
}

*Corresponding author: Tuan-Jen Fang, Department of Otolaryngology - Head \& Neck Surgery, Chang Gung Memorial Hospital \& Chang Gung University College of Medicine, Tao-Yuan, Taiwan, Tel: +886 3328 1200, ext: 9371; E-mail: fang3109@cgmh.org.tw

Rec date: Apr 29, 2014; Acc date: June 06, 2014; Pub date: June 08, 2014

Copyright: (c) 2014 Hsu YP, et al. This is an open-access article distributed under the terms of the Creative Commons Attribution License, which permits unrestricted use, distribution, and reproduction in any medium, provided the original author and source are credited.

\begin{abstract}
Kaposi's sarcoma is a frequently seen AIDS-related malignant neoplasm in the head and neck region, especially in the oral cavity, but is rarely described in the HIV-negative and immunocompetent individual. We present the iatrogenic Kaposi's sarcoma affecting soft palate which developed in a 53-year-old male and discuss their histological features and differential diagnosis.
\end{abstract}

Keywords: Kaposi's sarcoma; Oropharyngeal; Chemokines

\section{Introduction}

Kaposi's sarcoma (KS) was first described by dermatologist Moritz Kaposi more than a century ago [1]. It is a low-grade vascular tumor and its pathogenesis is closely related to human herpesvirus VIII (HHV-8) [2]. KS has high prevalence in immunocompromised patients, mainly in those with acquired immunodeficiency syndrome (AIDS) or transplant-associated immunosuppression.

KS is more commonly seen in men than women and have four clinical variants such as classic (Mediterranean), endemic (African), posttransplant, and epidemic or AIDS-associated [2,3]. Regardless of the different clinical course and prognosis, all four variants of KS share similar histolopathogical features and HHV-8 DNA can be found in most cases [3].

Primary KS in oral cavity of an immunocompetent patient (i.e. classic KS) is extremely rare and thus may be misdiagnosed as hemangioma, pyogenic granuloma or other benign vascular lesions clinically. We report classic KS originated from right soft palate of an immunocompetent Taiwanese and demonstrate it histopathological findings and the treatment outcome.

\section{Case Report}

A 53-year-old male was referred to our otolaryngology outpatient department due to a soft palatal mass lesion found from local clinic incidentally. He denied recent fever and had no underlying history of diabetes mellitus, hypertension, denied routinely taking any medication or oral supplement. The patient mentioned about oropharyngeal wound in the childhood. The family members of him do not have history of HHV-8 infection.

Physical examination revealed a reddish-to-purplish, lobulated and firm lesion over right supratonsillar fossa, sized approximately $2 \times 2 \mathrm{~cm}$ (Figure 1). No cervical lymphadenopathy was palpated. According to his history of present illness and physical findings, the initial impression of this lesion was a benign lesion, favorably a hemangioma.

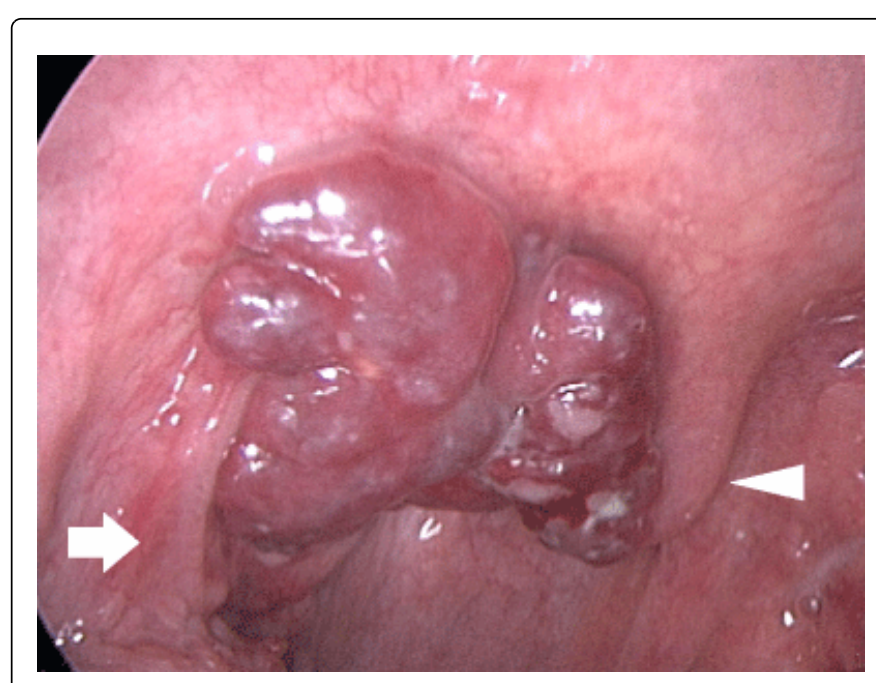

Figure 1: A reddish-to-purplish, lobulated and firm lesion located on the right side of soft palate. Arrowhead: uvula, Arrow: right tonsil

Transoral en bloc resection of this tumor was performed under general anesthesia was attempted and significant bleeding of the tumor was encountered during the operation. The postoperative recovery was smooth without oral wound bleeding. Histologic examination showed an ill-defined tumor composed of spindle cells separated by slit-like vascular spaces. The spindle cells showed only mild nuclear atypia (Figure 2). Immunohistochemial stain showed positive for HHV-8 that comfirms the diagnosis of Kaposi's sarcoma with margin involved. Serological tests for HIV, and Ebstein-Barr Virus were both negative. Magnetic resonance imaging (MRI) of the head and neck and Positron Emission Tomography (PET) scan only suggested postoperative inflammatory change at oropharyngeal area without definite evidence of cervical or distal metastasis. Due to positive margin, radiotherapy was given at total dose of 4000 cGy in 20 
fractions to the involved head and neck field. The patient remained free of disease after one year of follow-up.

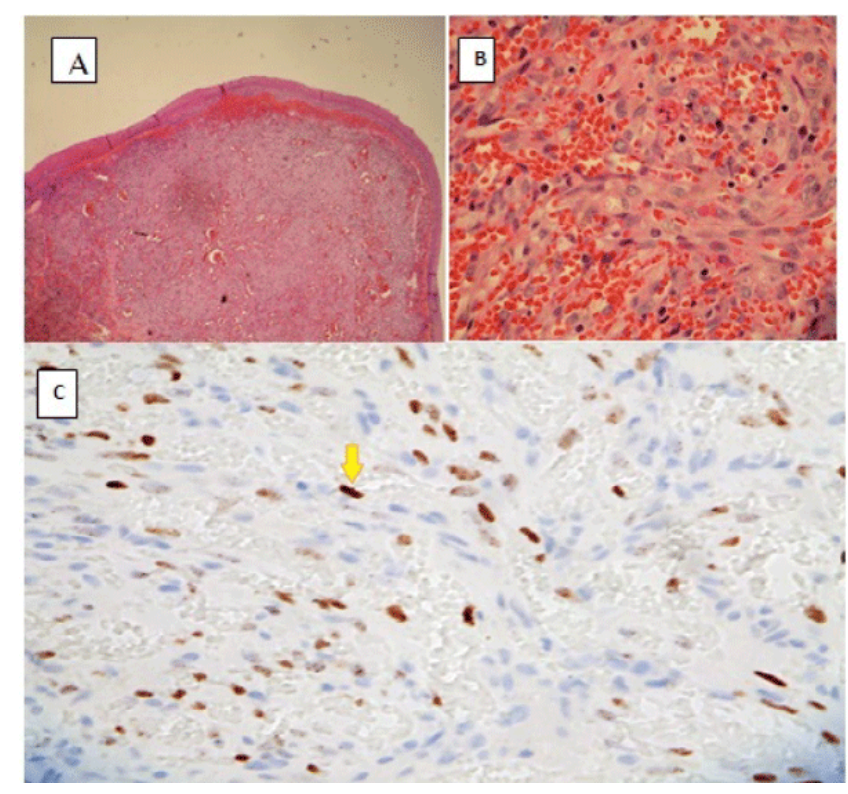

Figure 2: Histologic features of Kaposi's sarcoma in the nodular stage (hematoxyline-eosin stain). A. The dermis is expanded by a solid tumor nodule (original magnification 40X). B. Fascicles of relatively monomorphic spindled cells, with slit-like vascular channels containing erythrocytes (original magnification 400X). C. The nuclei of the tumor cells (yellow arrow) demonstrate immunoreactivity for HHV-8 (LNA-1 immunohistochemical stain)

\section{Discussion}

Kaposi's sarcoma is a malignant vascular neoplasm that currently known to be closely related to immunosuppressive state such as AIDS and post transplantation [2-4]. The relation of KS with infectious agent was established by Chang et al. [5] who demonstrated herpesvirus-like DNA sequences in AIDS-associated KS tissue [6]. Then subsequent studies suggested that the infection of Kaposi's sarcoma-associated herpesvirus (KSHV, also known as HHV-8), contributed to the tumorigenesis from disrupting the control of cellular proliferation, secreting chemokines that may activate angiogenesis and inhibit the immune type 1 helper-T-cell responses [3].

Although KS in the oral cavity is a common finding in HIV-positive patients, it is considered rare in non-HIV-infected individuals [7]. Oral KS that occur in patients with AIDS are called AIDS-associated (endemic) KS [2]. In our case, the sporadic occurrence is categorized as classical KS. It is a rare occurrence in immunocompetent individuals, presenting as firm, purplish-blue or reddish-brown plaques, papules or nodules originating from hands and feet and may involve the viscera or mucosa [8]. Classical KS is mostly seen in elderly of Mediterranean or Jewish decent [9].

KS lesions could be devided into 3 distinctive stages: the patch, plaque, and nodular stages. These tumors may ulcerate and cause marked lymphedema. The patch stage is characterized by irregular, small, jagged vessels lined by mildly atypical endothelial surface.
Extravasated erythrocytes, hemosiderin, and plasma cells are frequently present. Plaque-stage KS contained the same element in the patch stage but more prominent spindle cell. Promontory sign which means protrusion of normal blood vessels into the neoplastic ones may be seen in these two stages [9]. The nodular stage presents rather dome-shaped or polypoid lesion by a predominant spindle cell component that is percolated by slit-like vascular spaces, small jagged vessels, extravasated red blood cells, plasma cells, and lymphocytes $[10,11]$. The nodular stage often heralds nodal and visceral involvement, particularly in the African and AIDS-associated variants [8].

The clinical differential diagnosis of KS includes bacillary angiomatosis (BA), pyogenic granuloma, and hemangiomas. BA is caused by infection with gram-negative bacilli of the Bartonella family, results from an opportunistic infection in immunocompromised individuals [5]. This lesion presents similar to KS on clinical examination, because both are multifocal, dome-shaped to polypoid, and red-to-bluish lesions $[4,12]$. The differentiation between BA and $\mathrm{KS}$ is the circumscription, the presence of epithelioid endothelial cells lining the vessels, the lack of a spindle cell component, and the identification of the causative bacilli on silver stains (WarthineStarry) in BA $[4,12]$. Pyogenic granuloma is a rapidly growing pink-to-red and soft nodules on the skin, or gingival or oral mucosa; it bleeds easily and is often ulcerated. It is considered a reactive lesion that sometimes may be self-limited [13]. However, in the histological analysis, pyogenic granuloma does not have a spindle cell component and eosinophilic globules. Oral hemagiomas may show more dilated vascular spaces, resembling a cavernous hemangioma. Microscopic examination easily distinguished of hemangioma from KS, because the latter present atypical endothelial and spindle cells, eosinophilic globules, and a conspicuous component of reactive plasma cells [4].

HHV-8 infection also occurs later in childhood and during adolescence, and through some form of nonsexual contact. For this patient, HHV-8 infection could be through oropharyngeal wound in the childhood. Classic KS can be treated with surgical resection or one of the local treatments, such as radiation therapy. Chemotherapy may be used for widespread skin lesions or for KS that is in the lymph nodes, the lungs, or the digestive tract. Our case was treated with surgery and radial therapy, and there is no evident local recurrence and no further lesions observed after one-year follow-up.

This is one of the few cases of classic KS in ethnic Chinese. Clinicians should be aware that classic KS might be one of the rare occurrences of vascular lesions from the oral cavity. The definitive surgical resection and adjuvant radiation therapy provided adequate tumor control for this patient.

\section{References}

1. Kaposi M (1872) Idiopathisces multiples Pigmentsarkom der Haut. Archiv fur Dermatologi und Syphilis 3: 265-273.

2. Iscovich J, Boffetta P, Franceschi S, Azizi E, Sarid R (2000) Classic kaposi sarcoma: epidemiology and risk factors. Cancer 88: 500-517.

3. Cathomas G (2000) Human herpes virus 8: a new virus discloses its face. Virchows Arch 436: 195-206.

4. Calonje E, Fletcher CDM (2000) Tumors of the Blood Vessels/ Lymphatics. (2ndedn), Diagnostic Histopathology of Tumors. Hong Kong, Churchill Livingstone.

5. Cline MS, Cummings OW, Goldman M, Filo RS, Pescovitz MD (1999) Bacillary angiomatosis in a renal transplant recipient. Transplantation 67: 296-298. 
Citation: Hsu YP, Hsin LJ, Fang TJ (2014) Oropharyngeal Kaposi's Sarcoma from an Immunocompetent Host: A Case Report. J Cytol Histol 5: 254. doi:10.4172/2157-7099.1000254

Page 3 of 3

6. Chang Y, Cesarman E, Pessin MS, Lee F, Culpepper J, et al. (1994) Identification of herpesvirus-like DNA sequences in AIDS-associated Kaposi's sarcoma. Science 266: 1865-1869.

7. Reis-Filho JS, Souto-Moura C, Lopes JM (2002) Classic Kaposi's sarcoma of the tongue: case report with emphasis on the differential diagnosis. J Oral Maxillofac Surg 60: 951-954

8. Antman K, Chang Y (2000) Kaposi's sarcoma. N Engl J Med 342: 1027-1038.

9. Friedman-Kien AE (1981) Disseminated Kaposi's sarcoma syndrome in young homosexual men. J Am Acad Dermatol 5: 468-471.
10. Radu O, Pantanowitz L (2013) Kaposi sarcoma. Arch Pathol Lab Med 137: 289-294.

11. Van der Waal I, Knuutila LJ (2005) Kaposi sarcoma. Pathology \& genetics. Head and neck tumours: 193-194.

12. López de Blanc S, Sambuelli R, Femopase F, Luna N, Gravotta M, et al. (2000) Bacillary angiomatosis affecting the oral cavity. Report of two cases and review. J Oral Pathol Med 29: 91-96.

13. Jafarzadeh H, Sanatkhani M, Mohtasham N (2006) Oral pyogenic granuloma: a review. J Oral Sci 48: 167-175. 